\title{
FILTRATIONS AND NOETHERIAN SYMBOLIC BLOW-UP RINGS
}

\author{
PETER SCHENZEL
}

(Communicated by William C. Waterhouse)

\begin{abstract}
For a one-dimensional prime ideal in a local Noetherian ring it is characterized when the symbolic blow-up ring is an algebra of finite type. More generally, for a filtration of ideals of a local Noetherian ring there is a necessary and sufficient condition for the corresponding Rees ring to be a Noetherian ring. Applications concern asymptotic prime divisors and the analytic spread.
\end{abstract}

1. Introduction and main results. Let $(A, M)$ denote a local Noetherian ring. For a prime ideal $P$ of $A$ define $S(P)=\bigoplus_{n \in \mathbb{Z}} P^{(n)} t^{n} \subseteq A[t], t$ an indeterminate, the symbolic blow-up ring of $P$. Here $P^{(n)}$ denotes the $n$th symbolic power of $P$. In the case of a regular local ring, R. C. Cowsik asked whether $S(P)$ is an $A$-algebra of finite type or, equivalently, a Noetherian ring; see $[\mathbf{H}]$. This was shown to be true for some prime ideals defined by monomial curves in affine 3-space by C. Huneke $[\mathbf{H}]$ and S. Eliahou $[\mathbf{E}]$. In general the answer is negative as shown by $\mathrm{P}$. C. Roberts [Ro] by an example which grows out of M. Nagata's counterexample to Hilbert's fourteenth problem; see [N]. Here we will show a partial positive criterion.

(1.1) THEOREM. Let $P$ denote a one-dimensional prime ideal of an unmixed (e.g. regular) local ring $(A, M)$. Then $S(P)$ is an $A$-algebra of finite type if and only if there is an integer $k$ such that $a\left(P^{(k)}\right)<\operatorname{dim} A$.

Here $a(I)$ denotes the analytic spread of an ideal $I$ of a local ring; see [NR] for the definition. The statement follows from a more general result. To this end let $I:\langle M\rangle=\bigcup_{n>1} I: M^{n}$. Define $R_{M}(I)=\bigoplus_{n \in \mathbf{Z}}\left(I^{n}:\langle M\rangle\right) t^{n}$, the Rees ring with respect to the filtration given by $F=\left\{I^{n}:\langle M\rangle\right\}$. In $\left[\mathbf{S}_{\mathbf{1}}\right]$ the author investigated when $R_{M}(I)$ is finitely generated resp. integral over $R(I)=\bigoplus_{n \in \mathbf{Z}} I^{n} t^{n}$. This is closely related to the problem when the maximal ideal $M$ is a member of a certain set of asymptotic prime divisors related to $I$; see $(3.1)$ and $\left[\mathbf{S}_{\mathbf{1}}\right]$ for the precise statements. One of our main results here is to determine when $R_{M}(I)$ is an $A$ algebra of finite type.

(1.2) THEOREM. For an ideal I of a local ring $(A, M)$ the following conditions are equivalent:

(i) $R_{M}(I)$ is a Noetherian ring.

Received by the editors August 27, 1986 and, in revised form, December 18, 1986.

1980 Mathematics Subject Classification (1985 Revision). Primary 13E05, 13A17; Secondary $14 \mathrm{M} 05$.

Key words and phrases. Noetherian filtration, Veronesean subring, asymptotic prime divisor, analytic spread, symbolic power, symbolic blow-up ring, Rees ring, Hilbert's fourteenth problem. 
(ii) There is an integer $k$ such that

$$
a\left(I^{k} \widehat{A}:\langle M\rangle+p / p\right)<\operatorname{dim} \widehat{A} / p \quad \text { for all } p \in \operatorname{Ass} \widehat{A} .
$$

(iii) $M \notin \bar{A}(I)$.

Here $\widehat{A}$ denotes the completion of $A$. For the definition of $\bar{A}(I)$ see $\S 3$. The main idea of the proof is a criterion for $R(F)$, the Rees ring associated to a filtration $F$, to be a Noetherian ring. To this end let $R^{(k)}(F)$ denote the $k$ th Veronesean subring of $R(F)$; see $\S 2$ for the precise definitions.

(1.3) THEOREM. For a filtration $F=\left\{I_{n}\right\}$ of ideals of a local ring $(A, M)$ the following conditions are equivalent:

(i) $R(F)$ is a Noetherian ring.

(ii) There is an integer $k$ such that $R^{(k)}(F)$ is a Noetherian ring.

Together with the finiteness result of $R_{M}(J)$ over $R(J)$ the previous theorem yields (1.2). Moreover, (1.3) is of some independent interest.

In $\S 2$ we shall prove (1.3). $\S 3$ contains the proof of $(1.2)$ and its relation to asymptotic prime divisors. In $\S 4$ we consider the applications concerning symbolic blow-up rings of prime ideals. A characterization corresponding to (1.1) for arbitrary prime ideals will appear in $\left[\mathbf{S}_{\mathbf{2}}\right]$. There are also applications to monomial space curves. In the terminology we follow $H$. Matsumura's textbook $[\mathbf{M}]$ and $\left[\mathbf{S}_{\mathbf{1}}\right]$.

2. Noetherian filtrations. Let $A$ denote a commutative Noetherian ring with $F=\left\{I_{n}\right\}$ a filtration of ideals of $A$, i.e., a family of ideals of $A$ with $I_{n} I_{m} \subseteq I_{n+m}$ for all $n, m$ and $I_{0}=A$. By $R(F)=\bigoplus_{n \in \mathbb{Z}} I_{n} t^{n} \subseteq A[t], t$ an indeterminate, we denote the corresponding Rees ring. If $R(F)$ is a Noetherian ring, $F$ is called a Noetherian filtration.

(2.1) Proposition. $F$ is a Noetherian filtration if and only if there is an integer $k$ such that $I_{n+k}=I_{n} I_{k}$ for all $n \geq k$. In this case $I_{n k}=\left(I_{k}\right)^{n}$ for all $n \geq 1$.

The proof of (2.1) is well known; see [Re]. It follows from the fact that $R(F)$ is a Noetherian ring if and only if $A$ is a Noetherian ring and $R(F)$ is an $A$-algebra of finite type.

For a given filtration $F=\left\{I_{n}\right\}$ and an ideal $I$ of $A$ define $F A / I$ the filtration $\left\{I_{n}+I / I\right\}$ of $A / I$. In the case of a local ring $(A, M)$ define $F \widehat{A}$ the filtration $\left\{I_{n} \widehat{A}\right\}$ of $\widehat{A}$, the completion of $A$. For an integer $k \geq 1$ define $R^{(k)}(F)$, the $k$ th Veronesean subring of $R(F)$, i.e.,

$$
R^{(k)}(F)=\bigoplus_{n \in \mathbb{Z}} I_{k n} t^{k n}
$$

Clearly $R^{(k)}(F) \cong R\left(F^{k}\right)$, where the filtration $F^{k}$ is given by $F^{k}=\left\{I_{k n}\right\}$. Next we give another characterization for $R(F)$ to be a Noetherian ring.

(2.2) PROOF OF (1.3). First note that (i) $\Rightarrow$ (ii) holds for every integer $k \geq 1$ as easily seen. In order to show (ii) $\Rightarrow$ (i) we may assume $A$ a complete local ring as follows by (2.1). For the proof of the statement it is enough to show that $R:=R(F)$ is a finitely generated module over $S:=R^{(k)}(F)$. In the first step let $A$ be a (complete) domain. We have the inclusion $S \subseteq R$ which is in fact an integral extension. As a complete local domain $A$ is a Nagata ring; see [M, p. 234]. 
Therefore, $S$ as an $A$-algebra of finite type is also a Nagata domain; see [M, p. 240]. Furthermore, the quotient field of $A\left[t, t^{-1}\right]$ is a finite extension of the quotient field of $S$. Hence, the integral closure of $S$ in $A\left[t, t^{-1}\right]$ is a finitely generated $S$-module. In particular, $R$ is a finitely generated $S$-module.

In the second step assume, Ass $A=\{p\}$, i.e., the zero ideal of $A$ is a primary ideal. Put $r=\min \left\{n \in \mathbb{N}: p^{n}=0\right\}$. We prove the claim by an induction on $r$. The case $r=1$ was shown in the first step. Let $r>1$. Then $P:=\bigoplus_{n \in \mathbb{Z}}\left(p \cap I_{n}\right) t^{n}$ is a prime ideal of $R$ with

$$
O: P=\bigoplus_{n \in \mathbb{Z}}\left(O: p \cap I_{n}\right) t^{n}
$$

as easily seen. Next consider the short exact sequence of $S$-modules $O \rightarrow O: P \rightarrow$ $R \rightarrow R / O: P \rightarrow O$. Furthermore we have the following isomorphisms:

$$
R / O: P \cong R(F A / O: p)
$$

and

$$
S / O: P \cap S \cong R^{(k)}(F A / O: p) .
$$

Since $\operatorname{Ass}(A / O: p)=\{p A / O: p\}$ and $\min \left\{n \in \mathbb{Z}: p^{n} \subseteq O: p\right\}<, r$ the induction hypothesis yields that $R / O: P$ is a finitely generated $S$-module. On the other hand, $O: p$ is a torsion-free module of finite rank over $A / p$ as easily seen. Therefore we may imbed $O: P$ into a finite direct sum of $R / P \cong R(F A / p)$ which is a finitely generated module over $S / P \cap S \cong R^{(k)}(F A / p)$ by the induction hypothesis. Therefore $O: P$ and $R / O: P$ are finitely generated $S$-modules. So, $R$ is also finitely generated over $S$ by the above short exact sequence.

In the final step let Ass $A=\left\{p_{1}, \ldots, p_{s}\right\}$ and $O=q_{1} \cap \cdots \cap q_{s}$ a reduced primary decomposition of the zero ideal in $A$. By the second step of the proof

$$
R / Q_{i} \cong R\left(F A / q_{i}\right), \quad Q_{i}:=\bigoplus_{n \in \mathbb{Z}}\left(q_{i} \cap I_{n}\right) t^{n},
$$

is a finitely generated module over

$$
S / Q_{i} \cap S \cong R^{(k)}\left(F A / q_{i}\right), \quad i=1, \ldots, s .
$$

Therefore $R / Q_{i}, i=1, \ldots, s$, is a finitely generated $S$-module. Since

$$
O=Q_{1} \cap \cdots \cap Q_{s}
$$

there is an injection

$$
0 \rightarrow R \rightarrow \bigoplus_{i=1}^{s} R / Q_{i}
$$

which proves that $R$ is a finitely generated $S$-module as required.

3. Asymptotic prime divisors. For an ideal $I$ of a local ring $(A, M)$ let us consider the filtration $F=\left\{I^{n}:\langle M\rangle\right\}$, where $I:\langle M\rangle$ is defined as in the first section. Put $R_{M}(I):=R(F)$. Then $R_{M}(I)$ is a ring extension of $R(I)=$ $\bigoplus_{n \in \mathbf{Z}} I^{n} t^{n}$, the ordinary Rees ring with respect to $I$. In $\left[\mathbf{S}_{\mathbf{1}}\right]$ it was shown when $R_{M}(I)$ is finitely generated resp. integral over $R(I)$. As the main point of this section we want to characterize when $R_{M}(I)$ is a Noetherian ring. To this end recall some notations. Let $A(I)$ denote the well-defined set Ass $A / I^{n}$ for large $n$. 
Put

$$
A^{\prime}(I)=\bigcap_{J}\left\{A(J): I^{n} \subseteq J \subseteq\left(I^{n}\right)_{a} \text { for some } n \in \mathbf{N}\right\}
$$

where $I_{a}$ denotes the integral closure of $I$.

(3.1) Proposition. For an ideal $I$ of $(A, M)$ the following conditions are equivalent:

(i) $R_{M}(I)$ is a finitely generated graded $R(I)$-module.

(ii) $a(I \widehat{A}+p / p)<\operatorname{dim} \widehat{A} / p$ for all $p \in \operatorname{Ass} \widehat{A}$.

(iii) $M \notin A^{\prime}(I)$.

Here $\widehat{A}$ denotes the completion of $A$. The analytic spread $a(I)$ of $I$ is defined by $a(I)=\operatorname{dim} R(I) / M R(I)$; see [NR]. For the proof of (3.1), see [ $\left.\mathbf{S}_{\mathbf{1}},(6.4)\right]$. For our purposes here we call a prime ideal $P \supseteqq I$ a sticky prime divisor of $I$ if $P \in A\left(I^{k}:\langle P\rangle\right)$ for all large $k$. The set of sticky prime divisors of $I$ is denoted by $\bar{A}(I)$. Clearly $\bar{A}(I) \subseteq A(I)$ and $\bar{A}(I)$ is a finite set. Note that $P \notin \bar{A}(I)$ for a minimal prime divisor $P \supseteqq I$.

(3.2) PROOF OF (1.2). In order to show (i) $\Rightarrow$ (iii) note that by (2.1) there is an integer $k$ such that

$$
I^{k n}:\langle M\rangle=\left(I^{k}:\langle M\rangle\right)^{n} \text { for all } n \geq 1 .
$$

That is, $M \notin A\left(I^{k}:\langle M\rangle\right)$ for a large $k$ and $M \notin \bar{A}(I)$ by the definition.

Next we prove (iii) $\Rightarrow$ (ii). By the assumption there is an integer $k$ such that $M \notin A\left(I^{k}:\langle M\rangle\right)$. Put $J=I^{k}:\langle M\rangle$. Then $J^{n}:\langle M\rangle=J^{n}$ for all large $n$. Therefore $R_{M}(J)$ is a finitely generated module over $R(J)$. Hence the conclusion follows by virtue of (3.1). In order to complete the proof let us show (ii) $\Rightarrow$ (i). By (3.1) we see that $R_{M}(J)$ is finitely generated over $R(J)$, where $J=I^{k}:\langle M\rangle$. Now it is easy to see that

$$
J^{n}:\langle M\rangle=I^{k n}:\langle M\rangle \text { for all } n \geq 1 .
$$

Therefore, we have an isomorphism $R_{M}(J) \cong R_{M}^{(k)}(I)$. Because $R_{M}(J)$ is finitely generated over $R(J)$ it is a Noetherian ring. Thus, $R_{M}^{(k)}(I)$ is also a Noetherian ring. Therefore, (1.3) yields that $R_{M}(I)$ itself is a Noetherian ring as required.

While $a(I)=a\left(I^{k}\right)$ for all $k \geq 1$ it is noteworthy to say that $a(I:\langle M\rangle) \neq$ $a\left(I^{k}:\langle M\rangle\right)$ for some $k$ as follows by the example (4.3)(1).

A particular case of the equivalence of (i) and (ii) in (1.2) has been shown by $\mathrm{A}$. Ooishi in [O].

(3.3) EXAMPLE. Let $K$ denote an infinite field. Take $3 m$-elements of $K$ which are independent over $K$ and denote them by $a_{i}, b_{i}, c_{i}, i=1, \ldots, m$. Then let $I_{i}=\left(a_{i} y-b_{i} x, a_{i} z-c_{i} x\right)$ and $I=\bigcap_{i=1}^{m} I_{i}$ in $A=K[x, y, z]_{(x, y, z)}$. It was shown by M. Nagata in [N] that if $m$ is a square $\geq 16$, then $R_{M}(I)$ is not Noetherian, i.e., $a\left(I^{k}:\langle M\rangle\right)=3$ for all $k \geq 1$.

4. Symbolic blow-up rings. For a prime ideal $P$ of a commutative ring $A$ put $P^{(n)}=P^{n} A_{P} \cap A, n \geq 1$, the $n$th symbolic power of $P$. Clearly $F=\left\{P^{(n)}\right\}$ defines a filtration of ideals of $A$. We define $S(P):=R(F)$ the symbolic blow-up ring with respect to $P$. In the case $A$ is a regular local ring R. C. Cowsik asked 
whether $S(P)$ is an $A$-algebra of finite type. This was answered in the negative by P. C. Roberts in [Ro]. He used Nagata's example (see (3.3)) in order to construct a prime ideal $P$ in $K[x, y, z]_{(x, y, z)}$ such that $S(P)$ is not Noetherian. Related to our investigations we prove the following criterion.

(4.1) COROLlaRY. Let $P$ denote a one-dimensional prime ideal of a local ring $(A, M)$. Then the following conditions are equivalent:

(i) $S(P)$ is an A-algebra of finite type.

(ii) There is an integer $k$ such that

$$
a\left(P^{(k)} \widehat{A}+p / p\right)<\operatorname{dim} \widehat{A} / p \quad \text { for all } p \in \text { Ass } \widehat{A} .
$$

(iii) $\widehat{A}(P)=\varnothing$.

ProOF. Because $P$ is a one-dimensional prime ideal it follows $P^{(n)}=P^{n}:\langle M\rangle$ for all $n \geq 1$ and $\bar{A}(P) \cong\{M\}$. Hence $R_{M}(P) \cong S(P)$ and (1.2) yields the statement.

In general, condition (ii) of (4.1) is not easy to control. Under some additional assumptions about the ring it simplifies as indicated in (1.1).

(4.2) ProOF OF (1.1). In the case $A$ is an unmixed local ring we have $\operatorname{dim} A=$ $\operatorname{dim} \widehat{A} / p$ for all $p \in$ Ass $\widehat{A}$. Furthermore, for an ideal of a local ring it is known that

$$
a(I \widehat{A}+p / p) \leqq a(I) \text { for all } p \in \text { Ass } \widehat{A}
$$

with the equality for at least one of them; see [NR]. Hence, (1.1) follows by (4.1).

(4.3) REMARKS. (1) Let $A$ be as in (3.3). Let $P$ denote the prime ideal with the generic point $x=t^{3}, y=t^{4}, z=t^{5}$. Then it was shown by C. Huneke in $[\mathbf{H}]$, resp. S. Eliahou in $[\mathbf{E}]$, that $S(P)$ is a Noetherian ring. In fact one can show that $a\left(P^{(2)}\right)=2$. So the claim follows by (1.1). For an application of (1.1) to the case of prime ideals defined by monomial curves in affine 3 -space see $\left[\mathbf{S}_{\mathbf{2}}\right]$, where $S(P)$ is shown to be a Noetherian ring for a large class of $P$.

(2) Let $A$ as above and $P$ the prime ideal constructed by P. C. Roberts in [Ro]. Then $S(P)$ is not Noetherian and therefore $a\left(P^{(k)}\right)=3$ for all $k \geq 1$.

(3) . Related to a generalization of Hilbert's fourteenth problem given by $O$. Zariski, in [Re] D. Rees showed that $S(P)$ is not a Noetherian ring for $P$ the prime ideal of a nontorsion point of an elliptic curve in the projective plane. Let $A$ denote the local ring at the origin of the coordinate ring of this curve. Then $\operatorname{dim} A=2$ and $a\left(P^{(k)}\right)=1$ if and only if $P^{(k)}$ is a principal ideal because $A$ is a normal ring. But this cannot be true since $P$ corresponds to a nontorsion point.

\section{REFERENCES}

[E] S. Eliahou, Courbes monomiales et algebre de Rees symbolique, Thèse, Université de Genève, 1983.

[H] C. Huneke, On the finite generation of symbolic blow-ups, Math. Z. 179 (1982), 465-472.

[M] H. Matsumura, Commutative algebra, 2nd ed., Benjamin, New York, 1980.

[N] M. Nagata, Lectures on the fourteenth problem of Hilbert, Tata Inst. Fund. Res., Lectures on Math., No. 31, Bombay, 1965.

[NR] D. G. Northcott and D. Rees, Reductions of ideals in local rings, Proc. Cambridge Philos. Soc. 50 (1954), 145-158.

[O] A. Ooishi, Noetherian property of symbolic Rees algebras, Hiroshima Math. J. 15 (1985), 581584 . 
[Re] D. Rees, On a problem of Zariski, Illinois J. Math. 2 (1958), 145-149.

[Ro] P. C. Roberts, A prime ideal in a polynomial ring whose symbolic blow-up is not Noetherian, Proc. Amer. Math. Soc. 94 (1985), 589-592.

[S1] P. Schenzel, Finiteness of relative Rees rings and asymptotic prime divisors, Math. Nachr. 129 (1986), 123-148.

[S $\left.\mathbf{S}_{\mathbf{2}}\right]$, Examples of Noetherian symbolic blow-up rings, Rev. Roumaine Math. Pures Appl. (to appear).

Sektion Mathematik Der Martin-Luther-Universität, DDR-4010 Halle, GerMAN DEMOCRATIC REPUBLIC 